\title{
General Characteristics of Crop Irrigation Model
}

\author{
Rae ZH Aliyev* \\ Erosion and irrigation Institute of ANAS, Azerbaijan \\ Received: 制: September 09, 2018; Published: 制 September 17, 2018 \\ *Corresponding author: RAE ZH Aliyev, Erosion and irrigation Institute of ANAS, Azerbaijan, Russia
}

\begin{abstract}
The analysis of the issues arising in the designation of the irrigation regime allows us to identify several main groups of factors affecting the election of the irrigation scheme, where the most important of them are: 1 . weather factors that determine the situation on the field and the conditions of growth;2. agrobiologic factors characterizing the properties of crops and its response to moisture conditions; 3 . soil-hydrological factors related to water regime and characteristics of root zone soil; 4.- Economic and commercial factors determining possible damage due to moisture conditions deviations from optimal conditions and costs of irrigation regime. However, it should be recognized, it carried the results of the analysis which showed that any successful methods of prediction of moisture plants focused on dynamics of soil moisture: maintaining soil moisture below not specified level, usually associated with the phases of the development of agricultural crops.
\end{abstract}

Keywords: The Task of Optimally-Operational Management; Water Resources; System Analysis; Mathematical Modules; Economic Criteria

\section{Introduction}

Irrigation of agricultural crops irrigation regime is a complex task that a variable is of great complexity owing to the large number of variables (the amount and frequency of rainfall, irrigation air temperature, soil temperature, air humidity, soil moisture, solar radiation, the reaction of plants to watering, etc.) and has a significant impact on the size and quality of the crop and on logistical costs incl. Moreover, the irrigation regime is the only one controlled variable, and one of the main and decisive factors determining getting planned crop yields, particularly in the zone of insufficient moistening [1]. It is therefore necessary, given the possibility of choosing options, find solutions to optimal from the point of view of the projected crop losses and the logistical costs: find the frequency and amount of irrigation, determining total schedule watering crops. Difficulty selecting rational irrigation schemes associated with a large number of diverse factors that must be taken into account when assigning the number of irrigations, their rules and deadlines. We must consider not only the current meteorological situation and its forecast the dynamics of soil moisture and water availability for plants, but also biological peculiarities of agricultural culture, the influence of irrigations on plants, productive-economic circumstances, as well as the relationship between taken at different points in time decisions on watered crops. [1.5, 6.7].

\section{Strokes: Study}

The basis for calculating the irrigation regime is a technique of evaporation calculation field. Optimize the use of water resources through the use of COMPUTERS is currently the most important trend of irrigated agriculture [2]. Research proven effective solution to the problem of optimal operational water management re quires the application of systems analysis, complex mathematical modules and economic criteria [1.5, etc.]. When the soil moisture model identification is based on data collected by area.

\section{Crop Irrigation Model Conforming to the Requirements of the Plants Must}

a. Describe the impact on productivity of the cultivated culture conditions of moisture throughout the growing period,

b. Describe the processes on the field at a level which allows to use vegetation data as experiments, and rich empirical material obtained in vivo [3],

c. Be simple enough, include the required number of parameters, each with specific physical meaning and quantify in vivo,

d. The model should be parametric "tough", i.e. the properties of the model should not change precipitously at small variations of parameters.

Humidity level for planting is defined as the need for plants in water, and the ability to use soil moisture, and if need be associated with meteorological conditions in the surface layer of air and with the magnitude of the aerial parts of irrigation opportunity for plants to use soil moisture is determined by the development of the root system and soil moisture [4]. In General, the estimated depth of wetting layer should be determined by the depth of penetration of roots of irrigated crops in the ground. Usually in practice irrigated farming irrigation conducted based soil soaking up to a depth of $1 \mathrm{~m}$, and patterns of water consumption for irrigation of fields are set for the soil layers, do not exceed the specified limit. [1.7] Irrigation sprinkler systems due to the low speed of seepage of moisture 
in soil irrigation norm is calculated from soaking the soil in 50-60 see. The current state of sowing in terms of its productivity in crop losses nevosstanavlivaemosti, caused the lack of moisture at any time, be useful to characterize the value expected at the time of $t$ harvest $\mathrm{U}(\mathrm{t})$.

Indeed, $\mathrm{U}(\mathrm{t})$ is the difference between the potential yield $\mathrm{U} 0$ (value, resulting in full needs seeding in water) and accumulation for $\left\{t_{n}, t\right\}$ yield losses due to suboptimal moisture inside of vegetation. Thus, for optimization problems moisture fields irrigated with minimum number of root zone moisture condition is the vector layer and the expected harvest [5]. As components of the vector of unregulated external influences $\mathrm{q}(\mathrm{t}$ ) must be greater than a whole meteorological parameter, affect the evaporation from soil surfaces and transpiration. Depending on the method of calculating this can be any combination of options: radiation balance, temperature and humidity, precipitation, and wind speed.

\section{It Is Believed that the Model of Irrigation System as a Whole is Inherent in the Following}

a. Intensity of flow processes in a vegetative organism within days changed. In doing so, due to the large inertia of the system soil-plant-atmosphere speed of change of biophysical processes does not correspond to the evolution of external factors impacting [3.5]. In this regard, in order to simplify the model vnutrisutochnye changes processes in the field and should not be treated per unit time scales take day.

b. Relation to the changing in the ontogenesis of sensitivity of plants to external factors Wednesday as part of an overall management system should operate irrigation scheme development forecast of crops [6]. The establishment of such a scheme requires the development of a fairly simple development model oriented to use in production environments. When you build a model of development must be borne in mind.

c. As an indicator characterizing the biological age of sowing, it is advisable to take phase plant development.

d. Plants observed sequence of phases of development, the latter permanently, and if in the body of the plant not come this phase, the next phase would not pass even if you have optimal conditions.

1. in the major factor influencing the pace of development of plants are thermal growing conditions of culture, characteristic of which is the average daily air temperature. The influence of other factors is small; the exception is the period before germination, where soil moisture plays a significant role [7]. However, at the beginning of vegetation planting in damp soil optimally determining factor is heat.

e. To undergo a plant next development phase for this phase requires constant amount of average daily temperatures ti, with ti and threshold temperatures are Permanent class, little dependent on temperature fluctuations within a period of $[1,8$, 10]. Using the above model metrics allows you to parameterize it on quite easily available in the archives of the results of the multi-year hydrometeorological observations over development of crops in real conditions in different climatic regions [8].

\section{Model of Crop Losses with a Lack of Moisture}

Model of crop losses, on the one hand, should include changes in the sensitivity of sowing to deficiency of moisture in different periods of vegetation, on the other hand reflect the different impact on planting of different levels of moisture. [2.3]

\section{Dependence of Crop Losses from Moisture Levels Must Meet the Following Main Provisions}

a) The loss in the regular water supply at any period of life of plants leads to delay carrying process, resulting in crop losses are absent only in smooth water throughout the growing season [9]. Yield losses depend on the duration of drought and it accumulates so that no favorable moisture conditions subsequently unable to obtain maximum yield corresponding to the level of farming and fertility soil.

b) Resistance of plants in ontogenesis in relation to the lack of moisture varies in time, and therefore the impact of this shortage of on the final harvest is fickle and has a maximum at a critical time, with a critical period in relation to the air drought coincides with a critical period to drought soil. Found that a critical period in the life of the plant begins since reduktsionnogo the parent cell and continues to the flowering and fertilisation, inclusive [1.2,4]. For one sunset factors-conditions of moisture-naturally assume that crop losses depend on the intensity of moisture deficit:

$$
U(t)=d U / d t=-\beta(\lambda, t) \cdot U
$$

Where, $(\beta)(\lambda, t)$ is the relative loss in crop when moisture $(\lambda)$ at the time of $t$. In equation (1) implies that at the time of loss $t$ defined only by the current state $U(t)$ and do not depend on the background. Of the impact that lack of moisture at different points in the ontogeny of plants remains unchanged [1.3]. On the other hand, the degree of this impact is determined by the biological age of the plants, the same lack of moisture at different points of vegetation leads to different ways to absolute value fall harvest. This allows you to accept the hypothesis of razdelimosti arguments to functions $\beta$ and write:

$$
\beta(\lambda, t)=g_{1}(t) \cdot g_{2}(\lambda)
$$

Function $(\mathrm{g}) 1(\mathrm{t})$ is determined by the combination of internal physiological and genetic factors characterizing features of cultivated crops. (g) $1(\mathrm{t})$ shows how changes in the ontogenesis of sensitivity of plants to the lack of moisture. Function (g) $2(\lambda)$ adjusts the losses according to the physical moisture $(\lambda)$, i.e. defines the dependence of crop losses in the fixed point of the level of moisture. In the range of available moisture availability is fickle. ranging from the smallest capacity W NV to moisture the capillary break W RK moisture availability changed little. Ranging from W RK to the wilting point moisture $\mathrm{W} w$ the availability of moisture causes gradual- 
ly increasing violation of most of the processes associated with the growth of plants. The lower limit of the optimum humidity is in the range of the humidity of the capillaries break, while humidity ending growth corresponds to the wilting point moisture. For certainty is taken line-character (g) $2(\lambda)$, then the equation (1) becomes:

$$
U(t)=-g_{1}(t) \cdot(1-\lambda) \cdot U
$$

Many practically important cases of irrigation mode creates such conditions of moisture, that $\mathrm{U}(\mathrm{TK}) \approx \mathrm{U} 0$,

Where to harvest (end of vegetation); U 0 -humidity level for sowing, during vegetation does not restrict growth processes. At this level (3) can be replaced.

$$
U(t)=-g_{1}(t) \cdot(1-\lambda) \cdot U_{0}
$$

So, $(\beta)$ describes one of the most important parties of the system-the ability to counter adverse external factors(Figure 1).

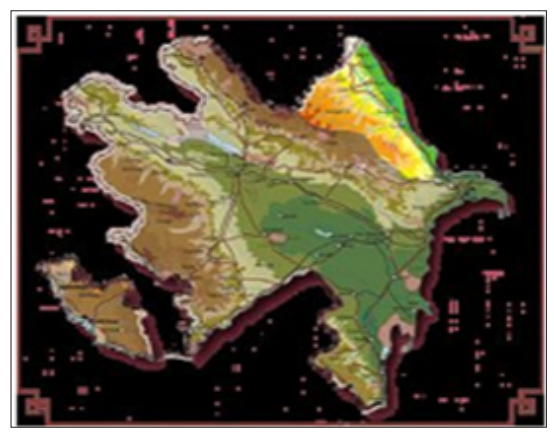

Figure 1.

\section{Moisture Exchange Process Model}

The proposed method of calculating soil moisture dynamics is based on the set of meteorological factors on the field, including radiation balance, temperature and humidity, wind. This method can be used successfully in a variety of soil and climatic conditions. When calculating the total evaporation (e)with its components are calculated separately-the physical evaporation (e)s and transpiration (e)t, because it does not indicate the total evaporation moisture seeding, but only the transpiration is productive by evaporation. To calculate the physical evaporation under conditions of sufficient moistening the soil surface expression is applied:

$$
E_{\phi}=b_{1} \Theta_{1} d+b_{2}\left(R_{0} \cdot e^{-n \infty}-B\right)
$$

where the adopted exponential attenuation of radiation, vegetation cover; (b) 1, (b) 2, $\theta 1$-coefficients, determined conditions of turbulent Exchange and depending on the average daily air temperature and leaf area; (d) -the average daily humidity deficit; -radiation balance on the surface vegetation;(n) $(\phi)$-absorption coefficient, which characterizes the velocity radiation balance reduction in vegetation cover; in-heat transfer in the soil [10]. As per diem amount of heat exchange in soil low (10-30 kcal/cm. d) and their relation to the daily amounts of radiation balance does not exceed a few percent, of often neglected, dependence (4) is used in fairly damp surface. Such soil condition is not lengthy, the soil dries up, leaving the physical evaporation becomes less than the maximum possible and defined by meteorological conditions. If soil moisture is different from optimal speed moisture evaporation suspended can be calculated by the following formula:

$$
E_{\phi}=j\left(W-W_{p x}\right)
$$

where (j) -aspect ratio, changing to different soils from 0.7 to $1.5 \mathrm{~mm} /$ day. W RK -humidity gap capillary connection in $\%$ of the volume of soil, if evaporation measured in mm/day. W -soil moisture. Because when $\mathrm{W}<\mathrm{W}$ RK evaporation, dramatically decreasing does not cease and enters into the third phase, instead of W RK in equation (5) will be used by wilting point moisture $\mathrm{W}$ w . Because the magnitude of ef cannot be greater than the maximum possible physical evaporation in the General form:

$$
E_{\phi}=\left\{\begin{array}{l}
j\left(W-W_{3}\right), W \leq W_{k P} \\
E_{\phi 0, W>W_{k P}}
\end{array}\right.
$$

Where, $\mathrm{W}_{\text {кр }}=/ \mathrm{j}+\mathrm{W}_{3}$-critical humidity above which the soil does not limit evaporation and the latter is defined exclusively by meteorological conditions. By calculating (7) physical evaporation, potential transpiration etrhaving a place with unlimited access of moisture to the root system, [6] can be calculated by the formula:

$$
E_{m p}=b_{1} \theta_{2} d\left[R_{0}\left(1-\theta_{3} e^{-m \Phi \omega}\right)-\left(1-\theta_{3}\right) \cdot B\right]-\left(1-\theta_{3}\right) \cdot E_{\Phi}
$$

Where the coefficients $\theta 2$ and $\theta 3$ depends on the wind speed and the remaining leaf area designations are the same as in (5). Dependence of transpiration (e)t of soil moisture is non-linear. With a fixed value volatility function Em/ W, portrayed by curve. She is approaching with an increase in soil moisture to a critical W CT and tends to zero with a decrease in soil moisture to humidity sustainable initializing $\mathrm{W} \mathrm{w}$ :

$$
\begin{aligned}
& W \leq W_{3} \\
& W_{3}<W<W_{k m} \\
& W>W_{k m}
\end{aligned}
$$

Wilting point moisture is determined by the mechanical composition of soil and practically does not depend on the properties of plants. It characterizes the lower limit of available soil moisture. Water consumption slightly compared to the consumption in normal conditions of life and they can be neglected. [1, 4.8] In the work of the $\mathrm{B}_{\mathrm{g}}$ [2] Aliyev shows that increasing volatility reduces critical suction pressure and increases humidity W CT corresponding to the optimal consumption of plants. The dependence of W CT = - ( ) approximated by linear function

$$
W_{k m}=W_{k m}^{1}+K_{w} \cdot E_{n_{0}}
$$

Where is -initial critical humidity corresponding to soil moisture, slows down transpiration when small ; Tow -gain-factor of proportionality; , To w -depend mainly on water-physical properties of soils, (8) and (9) it is possible to calculate the indicator of water availability for sowing $(\lambda) \mathrm{t}$ when advanced terrestrial mass representing the ratio of transpiration of actual to potential transpiration. Given the dependence of the indicator $(\lambda) \mathrm{w}$ in the beginning of vegetation on soil moisture and given the nature of the soil moisture availability dependencies from its humidity (10), it is approximated as 


$$
\lambda_{w}=\left\{\frac{\frac{W-W}{3}^{W \leq W_{3}}}{W_{k}-W_{3}}{ }_{W>W_{k}} W_{3}<W<W_{k}\right.
$$

Moreover, the value of $\mathrm{W}$ to close to moisture the capillary break, W RK and the transition from $(\lambda) w$ to $(\lambda)$ t occurs when the value of the index sheet $w$ approximately equal to 1 . Soil moisture Dynamics calculations are simplified, if we use one or the other kind of bioclimatic method $[1,5,7]$. Photovoltaic method is based on the concept of biological water consumption curve, which represents the seasonal variations of water consumption under specified weather conditions. This curve corresponds to the biological needs of plants and is sustained characteristic of impact properties of plants for their water consumption under optimal soil vlagozapasah. When a known biological curve toG(t) actual water consumption with optimal vlagozapasah is calculated by the formula:

$$
E_{\Phi}^{0}=K_{G}(t) \frac{E_{o \Phi}(t)}{E_{c p}(t)}
$$

where $\mathrm{e}_{C P}$-srednesezonnyj progress in terms of volatility, which is calculated to $(\mathrm{G}) ;(\mathrm{E}) 0 \mathrm{f}$-actual evaporation. In the case where soil moisture is less than optimal for weather conditions and fixed data development phase, evaporation becomes less calculated using biological curve, and it should be 2, 4, 8] according to the formula: биологической кривой и его следует рассчитывать 2,4,8]

$$
\lambda=E_{\Phi} / E_{\Phi}^{0}
$$

$\mathrm{W}$ to -critical soil moisture. The value of moisture by the expression

$$
d W / d t=E_{\Phi}-E_{m}
$$

In the mezhpolivnoj period in the absence of precipitation, and with disregard of vodoobmennom korneobitaemyh soil layers with underlying layers of soil moisture change in time find expression

$$
d W / d t=E_{\Phi}-E_{m}
$$

where, (e)s and et find out (7) and (9). After watering, taking soil moistened evenly [2.3] and that humidity layer below the calculated beginning to change only after the current layer is saturated to the smallest capacity, when humidity preirrigation $\mathrm{W}_{\mathrm{g}}$ poslepolivnaja soil moisture $\mathrm{W} p$ is determined by the formula:

$$
W^{i}=W^{g}+ \begin{cases}0 & H=0 \\ H & 0<H<H_{B}-W^{g} \\ W_{H}-W^{g} & \end{cases}
$$

where, n-norm watering similarly affects soil moisture. Posleosadkovaja soil moisture estimated by the formula (5.49) with the replacement irrigation norm $n$ on precipitation amount n. Accounting for runoff and groundwater recharge in vegetation can be pro- duced by one of the known methods (15). The simplest way of accounting for the recharge of groundwater in the vegetation period is the introduction of podpravok, independent from both the depth of occurrence of groundwater and soil mechanical composition and type of crop. In [1, 2, 7, 8 etc.] the following income dependency groundwater in crop root zone:

$$
K_{c}=E / e^{m \cdot h_{2}}
$$

Where $\mathrm{g}$ is the groundwater flow, $\mathrm{mm} /$ day; E-evaporation in $\mathrm{mm} /$ day; (h) g -depth of groundwater; (m) -factor depending on soil properties and plant development phase; In (17) number of plants fresh groundwater $(G)$ when their closest occurrence is determined by:

$$
C=E(v) \cdot(g), m m
$$

where, (g) -groundwater utilization depending on depth and soil mechanical composition; (E) (v) -water consumption by crops, $\mathrm{mm}$.

$$
(E)_{v}=E \cdot T o_{G}
$$

where e is evaporation, mm.; (G) -photovoltaic evaporation coefficient of crops.

\section{References}

1. Alekperov KA (1980) Pochvoezionnaya map and land protection. Moscow

2. Aliev BH the problem of desertification in Azerbaijan and ways to solve it, Ziya-Naji publishing house Baku-2005

3. Zaslavsky MN (1969) To the draft classification of soils according to the degree of erosion. Sat. Materials on the methodology of soil-erosion mapping of erosion control measures.

4. Erosiology MN (1983) Zaslavsky, publishing house Higher School

5. Ibrahimov AA (2001) Mapping of eroded soils on agricultural lands, (on the example of the Dashkesan region of the Azeri SSR

6. Ibrahimov AA (2000) Agroecological feature of eroded soils of Azerbaijan. Materials on the study of erosion and irrigation and soil conservation in Azerbaijan. Baku

7. Sobelyov SS (1948) The development of erosion processes on the territory of the European part of the USSR and the struggle against them. Publishing House of the USSR Academy of Sciences, 1

8. Sobolev SS (1961) Protection of soils from erosion.

9. Filkov VA (1958) Soil-erosional zoning of the Kamensk and Rostov regions. Sb. Soil erosion and struggle with it, Moscow.

10. Jozefacik A, Jozefacik CZ (1995) Erozya aqroekosystemow. Panstwowa Inspekcya Ochrony Srodowicka. Warszawa: Putawwy as IUNG. 168.1995 
ISSN: 2574-1241

DOI: $10.26717 / B J S T R .2018 .09 .001745$

RAE ZH Aliyev. Biomed J Sci \& Tech Res

(c) (i) This work is licensed under Creative

Submission Link: https://biomedres.us/submit-manuscript.php

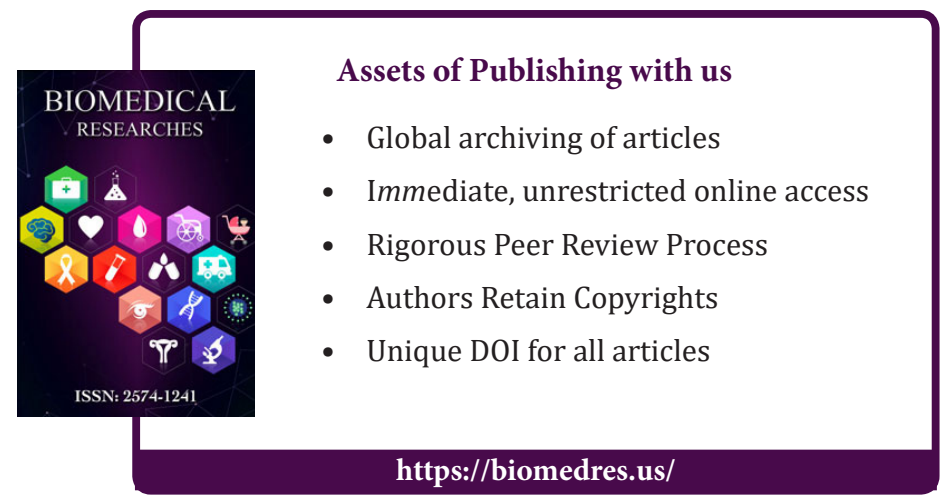

